\title{
Neonatal Pain Management among Nurses and Midwives at Two Kigali Hospitals
}

\author{
Claudine Muteteli $^{1 *}$, Olive Tengera ${ }^{1}$, Marcella Gowan ${ }^{1,2}$ \\ ${ }^{1}$ School of Nursing and Midwifery, University of Rwanda, College of Medicine and Health Sciences, Kigali, Rwanda \\ ${ }^{2}$ University of Illinois at Chicago, Linfield College, Good Samaritan School of Nursing, USA
}

*Corresponding author: Claudine Muteteli. School of Nursing and Midwifery, College of Medicine and Health Sciences, University of Rwanda, Remera Campus, 11 KG 47, Kigali, Rwanda. Email: muteteli84@gmail.com

\begin{abstract}
Background

The care of high-risk neonates in intensive care units is a relatively new field in resource-limited countries. Consequently, pain management of neonates has not been included or considered as part of neonatal care. Neonatal pain is more difficult to assess than adult pain, as they are unable to self-report. Nurses and midwives caring for neonates have a professional responsibility to recognise and manage neonatal pain.

Objectives

To assess the knowledge, attitude, and practice of nurses and midwives providing neonatal pain management at two hospitals in Kigali.

\section{Methodology}

A descriptive cross-sectional design was used. Data were collected from a convenience sample of 66 nurses and midwives providing neonatal care. Data analysis was achieved through descriptive and inferential statistics.

Results

The majority (74.2\%) demonstrated a low level of knowledge of neonatal pain and its management. Over half $(51.5 \%)$ had a positive attitude toward neonatal pain management, though over three quarters $(84.8 \%)$ reported a low level of integrating pain management into practice.

\section{Conclusions}

There is a knowledge deficit among the nurses and midwives providing neonatal pain management. A gap lies between their attitude and practice. More educational opportunities are needed to better assess and manage pain in the neonatal patient.
\end{abstract}

Rwanda J Med Health Sci 2019;2(2):138-146.

Keywords: Neonate, NICU, pain management, knowledge, attitude, practices

\section{Background}

Hospitalised neonates experience pain from both routine procedures and medical interventions many times a day. [1] In the past, it was thought neonates do not experience pain due to their physical immaturity.[2] Today we know that neonates experience measurable physiologic and behavioural responses to pain, as demonstrated in shortterm complications, as well as neurologic impairments leading to long-term sequelae.[1,3,4] Also, neonatal pain can be a barrier to parental bonding.[5] It has been shown that both term and preterm infants hospitalised in Neonatal Intensive Care Units (NICUs) experience on average 12 painful medical procedures per day which are mainly left untreated.[6]

There is increasing advocacy for the right of newborn patients also to receive safe, and effective pain relief. Neonatal pain management has not been part of the plan of care in resource-limited countries where the focus has been solely on saving the lives of neonates in need. Since care of preterm and high-risk neonates in the NICU is a new field, some providers may not know, or believe, that neonates experience pain. [7] There is still controversy concerning if and when a fetus experiences pain, though research findings now suggest a fetus may experience pain as early as at 20 weeks gestation. [8] Furthermore, the evidence shows that preterm neonates are more sensitive to pain due to a lower threshold and marked hyperinnervation in their central nervous systems.[3]

As a result, preterm neonates with a prolonged NICU stay, experience the most exposure to painful stimuli, [3,6] which should be an essential nursing care consideration. Pain management interventions by the nurse in the neonatal care setting include:[7] 
1. Assessment of comfort;

2. Observation of response to the procedure;

3. Evaluation of effects on comfort and rest.

Additionally, acute pain in the neonate should involve both non-pharmacological and pharmacological techniques.[9] It is the professional responsibility, which includes ethical considerations, of nurses and midwives to be able to recognise and manage patient pain. This factor is even more so for the neonatal patient in which newborns unlike adults are not able to self-report and describe their pain.[10] Therefore, nurses and midwives must be knowledgeable of the assessment and management of neonatal pain and advocate for this vulnerable population.

There are few examples in sub-Sahara Africa (SSA) with evidence-based clinical practice (EBP) guidelines readily accessible on the hospital wards.[15] This finding no doubt would include the absence of pain management practice guidelines in pediatric or neonatal wards. Neonatal nurse experts associate the absence of practice guidelines with a knowledge deficit on the importance of neonatal pain and its need for management.[7,13,16] Knowledge, attitudes, and beliefs of nurses impact the effectiveness of pain management.[17] Knowledge deficits may also lead nurses to have resistant attitudes toward appropriate neonatal pain management.[13] Nurses and midwives are the most consistent neonatal caregivers; therefore, nursing assessments of pain and their related pain practices are of critical importance.

Several international studies,[11-13] reported that pain in neonates is often not recognised and undertreated. There are very few studies in Rwanda on patient pain management, and there were no published studies on neonatal pain in 2017 when this research was conducted. One Rwandan study showed that nurses in an adult intensive care unit lacked adequate knowledge and had a poor attitude towards pain management.[14]

This paper introduces the background and significance of neonatal pain management. The short-and long-term complications of failing to recognise and relieve neonatal pain are presented. The right of the newborn patient to receive safe and effective pain relief is reviewed. Nurses and midwives are presented as the most consistent neonatal caregivers and how their knowledge, attitudes, and clinical practices are of critical importance to this high-risk population. This study aimed to assess the knowledge, attitude, and practice of nurses and midwives concerning neonatal pain and its management in two neonatal hospital wards in Kigali, Rwanda.

\section{Methods}

\section{Design}

The study was a descriptive, cross-sectional design and conducted in the neonatal and pediatric wards of a public district hospital and a university referral hospital in the capital city of Kigali Rwanda. The study sites were selected for the high volume of preterm, and high-risk neonates cared for by the nurses and midwives. The sites were also capable of providing diagnostic tests and procedures. Data collection occurred from 1 February to 31 May 2017.

\section{Participants' recruitment}

Registered nurses and midwives working in the neonatal and pediatric wards of the two study hospitals and available to participate were recruited. A convenience sampling strategy was used from the entire population of 75 nurses and midwives working on the two selected hospital wards. There were three eligible participants excluded as they were on annual leave; however, 72 registered nurses and midwives were given the questionnaire, and 66 participants filled it out and returned it for data analysis. The sample size was then 66 participants.

\section{Measures}

The data were collected using a self-administered questionnaire that was adapted from a valid and reliable instrument used by Akuma and Jordan.[12] Permission to adapt and use the questionnaire was provided by the corresponding lead author,[12] via email on $26^{\text {th }}$ August 2016.

The instrument was composed of two sections including demographic characteristics, and the participant's level of knowledge, attitude, and practice.

Section 1: Sociodemographics (7 items); gender, profession, work experience (years), hospital unit, and pain management training. Response: Scored Yes (1) or No (0).

Section 2: Part A. Knowledge of pain management (11 items). All variables were scored using the McDonald's criteria,[18] with pain management as the outcome. Response: True/False. Scored correct answer (1 point) and incorrect ( 0 points). Percentage scores were used to categorise knowledge into five levels. McDonald's criteria,[18] included very low $(<60 \%)$, low (60 to 69.9$)$, moderate (70 to $79.9 \%$ ), high (80 to $89.9 \%$ ), very high (90 to $100 \%$ ).

Part B. Attitude or perception of painful procedures (3items) Question - rank painful procedures using a rating scale. Response from 0 (no pain) to 4 (very painful). Scored as no pain (0) and others, somewhat painful, moderately painful, quite painful and very painful was by SPSS transformed into painful (1). Question - rate pain of neonate compared to the pain of adult. Response from less pain (0), to more pain (1). Scored as less pain (0) and some pain (1). Question -assess the attitude of using pharmacological or comfort measures. Responses ranged from never (0), to others, rarely, often, usually, and always were transformed into the use of analgesia/comfort (1). Definition of Positive 
attitude - believed comfort/ analgesia should be used for painful procedures (above the mean of 65). Definition of Negative attitude - believed comfort/ analgesia was not needed (below the mean of 65).

Part C. Practice - neonatal pain management (6 items) Questions - Response rated from never to always use in practice. Scored as never (0) and others, rarely, often, usually, always were transformed (1). Does unit have a pain assessment tool (Yes 1, No 0), and pain management policy or protocol (Yes 1, No 0).

List most common pharmacologic agents used for pain relief, in order from most common first. List most common non-pharmacologic agents used for pain relief, in order from most common first, scored with the correct answer ( 1 point) and incorrect ( 0 points). Percentage scores were used to categorise practice questions into five levels, included very low ( $<60 \%$ ), low (60 to 69.9 ), moderate (70 to $79.9 \%$ ), high (80 to $89.9 \%$ ), very high (90 to $100 \%$ ).[18]

The content validity was obtained from two neonatal colleagues and one neonatologist to assure that the content was designed to measure the desired outcomes. The results of Cronbach's calculation range were 0.650.90 , with an average of 0.76 . The English version of the questionnaire was translated into French and backtranslated into English for content accuracy. A pilot study of six participants from the referral hospital and four from the district hospital completed the questionnaire for feasibility purposes.

\section{Data collection}

Arrangements for the study were made with the appropriate study site management to address the nurses and midwives working with neonates at a staff meeting. After the investigator presented the information about the study, nurses and midwives were invited to participate. Interested participants were assured their information would be anonymous and those willing to participate signed the consent form. The questionnaire was prepared and printed in two versions, English and French and each participant chose their preferred language. They completed the questionnaires on site, which took about 30 minutes. Some of the participants submitted the completed self-reported questionnaire on the same day, while others returned the completed questionnaire at a later date.

\section{Data analysis}

The completed questionnaires were analysed using descriptive and inferential statistics. The variables were coded and entered into SPSS Statistics (version 21). Frequencies were calculated for the demographic variables. Chi-square and Fisher's exact tests were used to test the association between knowledge and practice, attitude and practice. Results with a p-value $<0.05$ were considered as statistically significant.

\section{Ethical considerations}

Approval was obtained from the University of Rwanda, College of Medicine and Health Science Institutional Review Board and both study sites.

\section{Results}

Seventy-two nurses and midwives were approached to be in the study and 66 returned completed questionnaires for a response rate of $91.6 \%$. The findings indicated that the majority of nurses and midwives were female $(87.9 \%)$ and registered nurses $(92.4 \%)$ (table 1$)$. The majority $(72.2 \%)$ worked at the referral hospital; all worked with neonates (birth to 28 days). Almost two thirds $(65.2 \%)$ worked with neonates on the pediatric ward and over a third (34.8\%) worked in NICUs. About a third $(34.5 \%)$ had work experience of five to ten years, and more than a third (39.4\%) reported working less than five years. The majority $(71.2 \%)$ reported that they had not received training in neonatal pain management.

\section{Table 1. Sociodemographic Characteristics}

Sociodemographics n (\%)

\section{Gender}

Female

Male

Professional role

Registered Nurse $\quad$ 61(92.4)

Register Midwife

Hospital Level

Referral 48(72.2)

District 18(27.3)

Work Unit

Neonatal 23(34.8)

Pediatric 43(65.2)

Work experience (years)

$<5$

36(34.5)

$10 \quad 4(6.1)$

Neonatal pain education*

Yes 19(28.8)

No 47(71.2)

Neonatal pain training**

Yes 11(16.7)

No 55(83.3)

* Neonatal pain education provided in nursing school ** Neonatal pain in-service training at work

The participants were then asked a series of questions related to knowledge of neonatal pain management and the correct answers (true/false) with the attendant scores are presented (table 2). An *(asterisk) identifies the correct answer. The majority correctly answered 
the following false statements: neonates, especially preterm, do not experience pain $(80.3 \%)$; and that pain management is not as essential as the care of neonates because they do not remember painful experience (92.4\%). The majority also correctly answered the following true statements: neonates of 37 weeks to term can perceive pain $(93.9 \%)$, and neonates from birth to 28 days can perceive pain $(95.5 \%)$.

\section{Table 2. Responses related to Knowledge of Neonatal Pain Management $(n=66)$}

\begin{tabular}{|c|c|c|}
\hline & \multicolumn{2}{|c|}{ Response } \\
\hline & $\begin{array}{l}\text { True } \\
\mathrm{n}(\%)\end{array}$ & $\begin{array}{l}\text { False } \\
\mathrm{n}(\%)\end{array}$ \\
\hline $\begin{array}{l}\text { Neonates, especially preterm, do not experience pain. } \\
\text { Pain response is congenital. } \\
\text { Neonates experience pain less than adults. } \\
\text { Pain management is not essential to neonate } \\
\text { as they do not remember painful experiences. } \\
\text { Physiologic stress related to pain can be more } \\
\text { dangerous than analgesia side effects. } \\
\text { Analgesia is too dangerous to use in neonates. } \\
\text { Neonates pain decreases quicker than adults. } \\
\text { Neonates need less analgesia than adults (per kg). } \\
\text { Neonates at } 26 \text { weeks have all physiological } \\
\text { requirements to perceive pain. } \\
\text { Neonates }<28 \text { weeks perceive pain. } \\
\text { Neonates } 28-36 \text { weeks perceive pain. } \\
\text { Neonates } 37 \text { weeks to term perceive pain. } \\
\text { Neonates birth to } 28 \text { days of perceives pain. }\end{array}$ & $\begin{array}{l}13(19.7) \\
41(62.1)^{*} \\
23(34.8) \\
5(7.6) \\
20(30.3)^{*} \\
24(36.4) \\
38(57.6) \\
56(84.9) \\
20(30.3)^{*} \\
43(65.2)^{*} \\
61(92.4)^{*} \\
62(93.9)^{*} \\
63(95.5)^{*}\end{array}$ & $\begin{array}{l}53(80.3)^{*} \\
25(37.9) \\
43(65.2)^{*} \\
61(92.4)^{*} \\
46(69.7) \\
42(63.6)^{*} \\
28(42.4)^{*} \\
8(12.1)^{*} \\
46(69.7) \\
23(34.8) \\
5(7.6) \\
4(6.1) \\
3(4.5)\end{array}$ \\
\hline
\end{tabular}

* Correct response

Based on the five levels of knowledge, the findings revealed the majority (74.24\%) had low knowledge, nearly a quarter $(22.73 \%)$ had moderate knowledge and a few $(3.03 \%)$ had high knowledge (figure 1$)$. Not one participant had a very low level or very high level of knowledge.

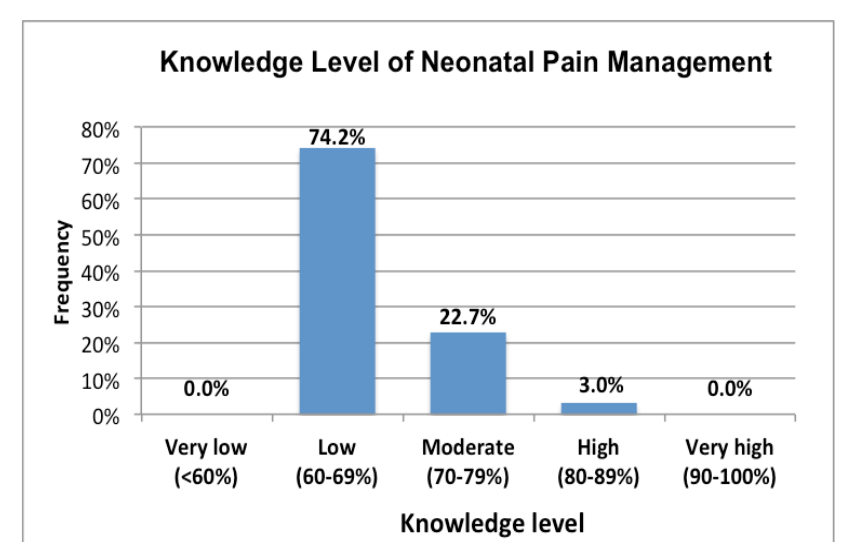

Figure 1. Level of knowledge of neonatal pain management

Knowledge of signs and symptoms of neonates experiencing pain

Participants were asked for signs and symptoms of neonatal pain (figure 2). The majority responded with crying $(83.3 \%)$, change in vital signs (56.15), and agitation or irritation $(53 \%)$.

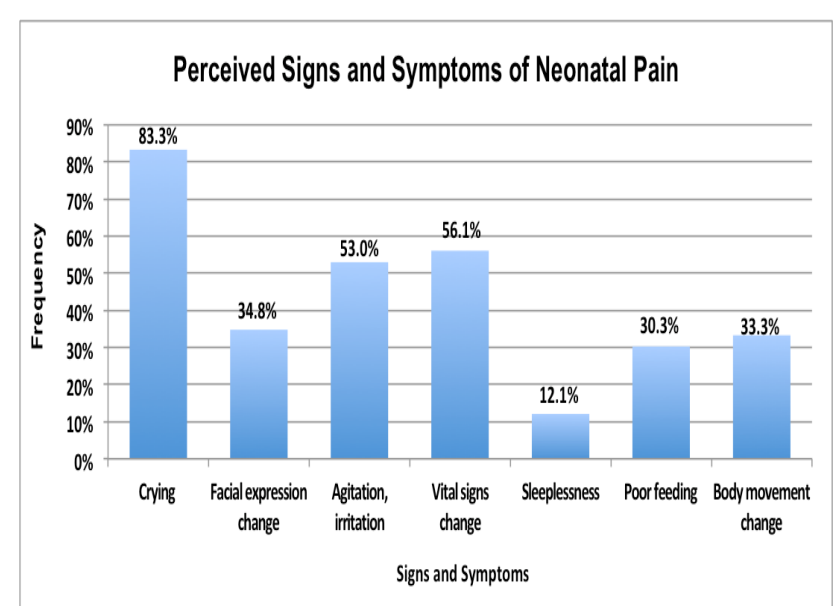

Figure 2. Perceived signs and symptoms of neonatal pain

Participants were asked to rate their perception of what they considered to be painful procedures for neonates, and the results were summarised using the Likert scale from no pain to very painful procedures (table 3). 
Table 3. Nurses' and Midwives' Perception of Painful Procedures for Neonates

\section{Neonatal Procedures}

\begin{tabular}{llllll} 
& $\begin{array}{l}\text { No } \\
\text { Pain } \\
\mathbf{n}(\mathbf{\%})\end{array}$ & $\begin{array}{l}\text { Some } \\
\text { Pain } \\
\mathbf{n}(\mathbf{\%})\end{array}$ & $\begin{array}{l}\text { Moderate } \\
\text { Pain } \\
\mathbf{n}(\mathbf{\%})\end{array}$ & $\begin{array}{l}\text { Quite } \\
\text { Painful } \\
\mathbf{n}(\mathbf{\%})\end{array}$ & $\begin{array}{l}\text { Very } \\
\text { Painful } \\
\mathbf{n}(\mathbf{\%})\end{array}$ \\
\hline Heel prick & $0(0)$ & $20(30.3)$ & $18(27.3)$ & $8(12.1)$ & $20(30.3)$ \\
IM injection & $8(12.1)$ & $20(30.3)$ & $13(19.7)$ & $25(37.9)$ & $0(0)$ \\
Catheter insertion* & $1(1.5)$ & $0(0)$ & $29(43.9)$. & $22(33.3)$ & $14(21.2)$ \\
Lumbar puncture & $0(0)$ & $2(3.0)$ & $0(0)$ & $17(25.8)$ & $47(71.2)$ \\
NGT insertion & $3(4.5)$ & $17(25.8)$ & $32(48.5)$ & $3(13.6)$ & $5(7.6)$ \\
Suctioning+ & $6(9.1)$ & $15(22.7)$ & $28(42.4)$ & $12(18.2)$ & $5(7.6)$ \\
ET intubation & $3(4.5)$ & $2(3.0)$ & $7(10.6)$ & $9(13.6)$ & $45(68.2)$ \\
Tape removal & $5(7.6)$ & $29(43.9)$ & $24(36.4)$ & $6(9.1)$ & $2(3.0)$ \\
Long line insertion & $0(0)$ & $1(1.5)$ & $13(19.7)$ & $22(33.3)$ & $30(45.5)$ \\
Chest drain & $1(1.5)$ & $0(0)$ & $1(1.5)$ & $3(4.5)$ & $61(92.4)$ \\
& & & & &
\end{tabular}

*Peripheral; +Upper airway; ^Adhesive

Participants perceived some procedures painless for neonates, including IM injections (12.1\%), upper airway suctioning (9.1\%), NG tube insertions (4.5\%), ET tube intubation (4.5\%) and removal of adhesive tape $(7.6 \%)$. Whereas, many perceived that chest drainage $(92.4 \%)$, lumbar puncture $(72.1 \%)$, ET tube intubation (68.2\%), long-line insertion (45.5), and heel prick $(30.3 \%)$ very painful procedures for the neonate.

Participants were asked to compare the painful procedures for neonates and adults. During a heel prick, some participants $(16.7 \%)$ reported there would be less painful for the neonate, nearly half (47.0\%) suggested it would be the same amount of pain, while a third $(36.4 \%)$ thought more pain for the neonate. Other procedures that the majority thought would be more painful for the neonate included, IM injection $(53.0 \%)$, peripheral catheter insertion $(54.5 \%)$, lumbar puncture 47(71.2\%), endotracheal intubation (72.7\%), long line (57.6\%), and chest drainage (83.3\%). Many others thought procedures such as nasogastric tube insertion (42.2\%), upper airway tube insertion (53.0\%), and adhesive tape removal (47.0\%), would elicit the same amount of pain as adults.

The attitude also rated how often they believed painful procedures should be performed using pharmacological interventions (analgesia) and non-pharmacological interventions (comfort measures) (figure 3). The questions were categorised and scored into two levels, either positive or negative attitude to pain management.

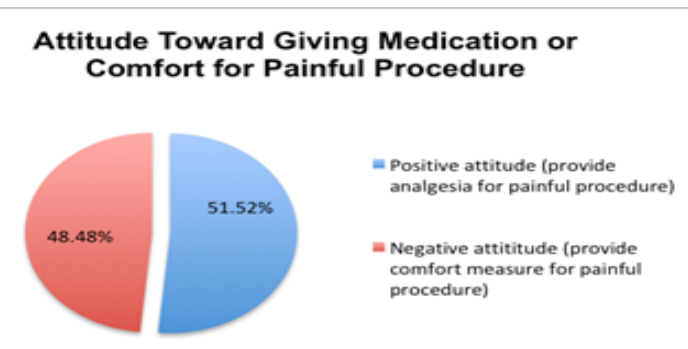

Figure 3. Attitude toward giving medication or comfort measures
The results showed that the majority $(51.52 \%)$ had a positive attitude toward providing pharmacologic intervention, or at minimum comfort measures, for painful procedures. The majority also reported that there was no neonatal pain management protocol $(93.94 \%)$ or pain assessment tool $(84.85 \%)$ available on their neonatal unit. The practice of neonatal pain management was assessed by asking what methods and how often they used nonpharmacological techniques (figure 4). Participants were asked when they use comfort / non-pharmacological measures for neonatal pain relief.

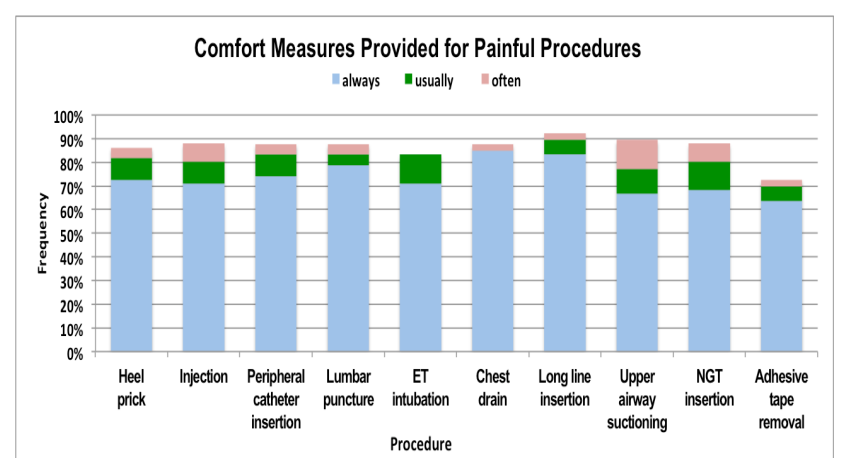

Figure 4. Comfort Measures Used for Painful Procedures

The most common comfort measures included breastfeeding $(31.8 \%)$ positioning $(24.2 \%)$, non-nutritive sucking $(12.1 \%)$, developmental care $(12.1 \%)$, and none $(13.6 \%)$. Others included sucrose $(3.0 \%)$, KMC $(1.5 \%)$, swaddling (1.5).

Participants were asked when they use pharmacological measures for neonatal pain relief (figure 5). The majority used analgesia for a lumbar puncture $(62 \%)$, ET intubation $(82 \%)$, chest drain $(80 \%)$, and long line insertion (50\%)(figure 5). Over half do not or rarely use comfort measures for heel prick $(98 \%)$, injection $(96 \%)$, peripheral catheter insertion $(96 \%)$, upper airway suctioning $(80 \%)$, NG tube insertion $(82 \%)$, or adhesive tape removal $(84 \%)$. 


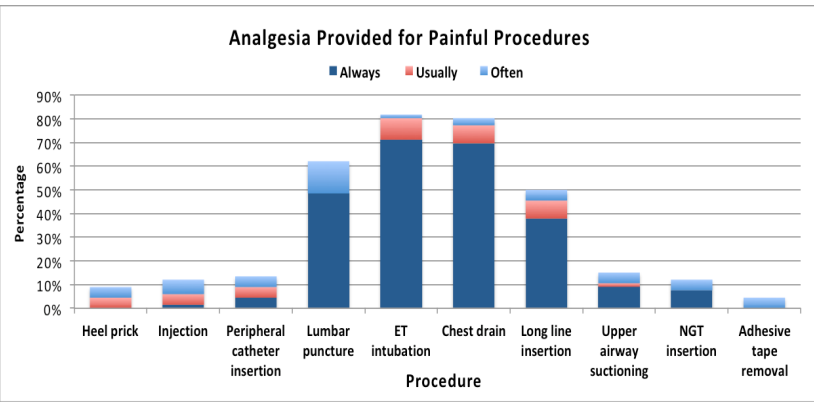

Figure 5. Analgesia Provided for Painful Procedures

The most common pharmacologic agents for pain relief include paracetamol $(86.1 \%)$, morphine $(10.8 \%)$, and sucrose $(3.0 \%)$. The hypnotic medication Midazolam was used by one participant, whereby the neonate might forget about the painful procedure, and another participant used diclofenac, which is used in adults.

The majority (84\%) do practice neonatal pain management, though at the low level (figure 6).

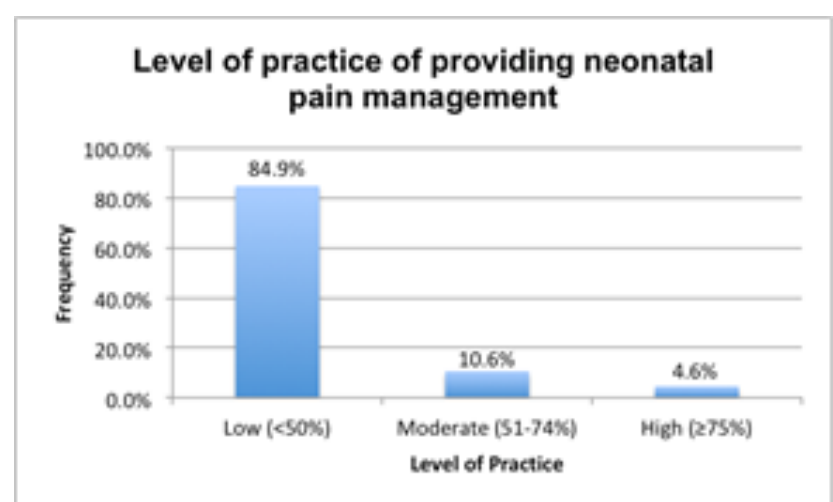

Figure 6. Level of practice of nurses and midwives providing neonatal pain management

There were significant associations between knowledge, attitude, and practice (table 4). The participants' knowledge was significantly associated with neonatal pain management practice $(p=0.03)$ and between the participants' attitude and practice $(\mathrm{p}=0.01)$.

Table 4. Association Between Knowledge and Practice, Attitude and Practice

\begin{tabular}{|c|c|c|c|c|c|c|c|}
\hline & \multicolumn{7}{|c|}{ Practice Level } \\
\hline & Low & Mod* & High & $x^{2}$ & $\mathrm{p}$ & Fisher's & $\mathrm{p}$ \\
\hline Knowledge level & & & & 14.8 & 0.03 & 10.4 & 0.03 \\
\hline Low & 43 & 6 & 0 & & & & \\
\hline Moderate & 12 & 1 & 2 & & & & \\
\hline High & 1 & 0 & 1 & & & & \\
\hline Attitude level & & & & 7.6 & 0.02 & 7.9 & 0.01 \\
\hline Negative & 30 & 0 & 2 & & & & \\
\hline Positive & 26 & 7 & 1 & & & & \\
\hline
\end{tabular}

*Moderate

\section{Discussion}

The majority of participants were female $(87.9 \%)$, registered nurses $(92.45 \%)$, working for 5-10 years in neonatal wards $(54.5 \%)$, indicating they were experienced and not novices managing neonates. Findings also showed that the majority did not receive any neonatal pain management education in school $(71.2 \%)$ or during their current employment $(83.3 \%)$.

The results showed that the majority $(74.24 \%)$ had a low level of knowledge of neonatal pain management at both facilities. This knowledge deficit may have an impact on the use of effective and optimal care of a neonate experiencing pain. This finding correlates with other studies,[21,22] which indicated that nurses had poor knowledge of neonatal pain management. In contrast, other studies, $[7,12,16,17]$ reflected higher scores.

The major areas of knowledge deficit in this study were related to the neonates' need for analgesia. The majority reported that neonates felt less pain, or the same amount of pain as an adult, which explains why the majority $(87.9 \%)$ thought that the neonate needs less analgesia than adults. The results indicated that the participants ranked chest drainage, lumbar puncture, and endotracheal intubation as very painful procedures; and adhesive tape removal, upper airway suctioning and NGT insertion as the least painful procedures. These findings are consistent with other studies. [7,11,12,16,23] The findings of low knowledge on neonatal pain management were likely related to the lack of formal teaching in nursing school and lack of continuing professional education in the hospital. Furthermore, the neonatal units lacked a pain management protocol or pain assessment tool. Neonates in pain need comfort measures or administration of analgesia, and nurses and midwives are in the optimal position to 
bring some relief. Effective pain management is an evidence-based standard of care for preterm and term newborns and may potentially improve their clinical and neurodevelopmental outcomes.[24]

The results indicated that about half of the participants had the right attitude to provide pain management. Almost all participants believed that neonates were able to perceive pain, similar to other studies, $[6,20,21]$ and that comfort measures should always be used. However, most participants believed that analgesia should never or rarely be given, indicating a negative attitude. This may imply that the participants were unaware of the safe use of analgesia, did not know how to give analgesia to neonates, or unsure of the physician's prescription.

The study findings concur with the literature that some nurses have a knowledge deficit of pharmacology and inadequate pain assessment skills which impacts their attitude and practice.[13,25] Nevertheless nurses who work with neonates have a responsibility to provide comfort measures as well as pharmacological treatment whenever a neonate is in pain. Nurses have a responsibility to provide comfort measures without waiting for an order, as it is the ethical thing to prevent harm. The goal for neonatal pain management ideally is to prevent pain, but if not, that pain should be managed by both non-pharmacological and pharmacological interventions that may even be more effective when they are used in combination.[4,24-26]

Understandably, if the knowledge and attitude to neonatal pain management were low, it makes sense the practice would also be low $(84.85 \%)$. The mean practice percentage was $52.08 \%$ with a range of $0 \%$ to $95 \%$. A study in Iran found similar results,[21] whereby pharmacological interventions were rarely used, or never used.[17,21] These findings contrast with the use of non-pharmacological interventions which participants reported as always used. These findings seem understandable given that the use of comfort measures is a fundamental role of nurses. However, the nurses' use of analgesia usually requires a doctor's prescription.

A very high percentage $(84.85 \%)$ reported that there were no pain assessment tools available, and (93.94\%) said there were no pain management policies or guidelines on the unit. This factor may explain the participant's low level of practice because it is recommended that for good neonatal pain management, every facility should have a validated pain assessment tool. Ideally, a neonatal pain control program should be in existence for providing routine assessment, minimising the number of painful procedures, preventing or treating pain from surgery and bedside invasive procedures and also for avoiding repetitive pain during newborn hospitalisation.[25,27] This result was similar to other studies.[3,6,21,23]

Not surprising, the most common pharmacological agent $(86.15 \%)$ used was paracetamol, as it is readily available and inexpensive, similar to other studies.[21,24]. The most common non-pharmacological comfort measures were breastfeeding (31.8\%), and positioning $(24.2 \%)$. In contrast, sweet solution $(68.6 \%)$ and nonnutritive sucking (58.8\%) were used in Bangladesh,[26] and oral glucose/sucrose $(68.6 \%)$ as the first common non-pharmacological measure used in Brazil.[17] Using a combination of various non-pharmacological measures achieves high effectiveness than when one of these measures is used alone.[25,28] Participants reported the use of sucrose (sugar) to relieve neonatal pain as never/ rarely by $81.82 \%$. This factor may shed light on understanding the low level of neonatal pain management practice revealed in this study. A 2016 Systematic Cochrane review that included 74 studies and 7,049 infants found that sucrose is safe and effective for minimising related pain procedures in newborns, [29] findings that were supported in other studies.[12,30,31]

Knowledge and attitudes toward pain management were both associated with pain management practices. This factor is consistent with the knowledge, attitude, and practice (KAP) model described by Chien-Yun and colleagues,[30] which suggested that knowledge and attitudes influence practices. The same findings were found in another study,[31] but another found no association between KAP.[26]

\section{Limitations}

This study was conducted in two specific hospitals in Kigali, Rwanda with a convenient sampling of the nurses and midwives so that results may be different at other sites. Due to the small sample size, these findings cannot be generalised to other populations. The data were based on the participants' self-report, which may not accurately reflect the actual clinical practice. Direct observation of the clinical procedures by the investigator may more accurately evaluate pain assessment and management in the NICUs and pediatric wards.

\section{Recommendations}

Nurses and midwives working with neonates need more educational opportunities to learn essential and ongoing pain management in nursing school and workplace facilities. This should also include the principles of palliative care.[28] The American Academy of Pediatrics recommends that all hospital units caring for neonates have a pain protocol, so nurses, midwives and other HCPs have ready access to EBP guidelines for the management of neonatal pain through both non-pharmacological and pharmacological techniques.[32] Neonatal units should also have neonatal pain routinely assessed using an objective context-specific pain management tool,[24] such as PIPP (premature infant pain profile) or NIPS (neonatal infant pain score). 


\section{Conclusion}

Results of the survey of nurses and midwives working with neonates showed there was a low level of knowledge and practice, though fairly positive attitude concerning neonatal pain management. There were significant positive associations between knowledge and practice, and attitude and practice. The implications of the findings are clear, in addition to the hospitals needing a pain management protocol including a pain assessment scale, nurses and midwives need regular training to better utilise pain management techniques and reduce unnecessary neonatal pain and suffering.

\section{Acknowledgements}

The authors are thankful to Dr Sue Prullage and Dr Pamela Meharry for their contribution to this manuscript.

This article is published open access under the Creative Commons Attribution-NonCommercial NoDerivatives (CC BY-NC-ND 4.0). People can copy and redistribute the article only for noncommercial purposes and as long as they give appropriate credit to the authors. They cannot distribute any modified material obtained by remixing, transforming or building upon this article. See https:// creativecommons.org/licenses/by-nc-nd/4.0/

\section{References}

1. Witt N, Coynor S, Edwards C, et al. A Guide to Pain Assessment and Management in the Neonate. Curr Emerg Hosp Med Rep 2016; 4: 1-10.

2. Lake SW. Barriers to Effective Pain Management in Preterm and Critically Ill Neonates. University of Kentucky, http://uknowledge.uky.edu/nursing_etds/7 (2013).

3. Grunau RE. Neonatal pain in very preterm infants: long-term effects on brain, neurodevelopment and pain reactivity. Rambam Maimonides Med J; 4. Epub ahead of print 2013. DOI: 10.5041/rmmj.10132.

4. Wilson-Smith E. Procedural pain management in neonates, infants and children. Br J Pain 2011; 5: 4-12.

5. Palomaa A, Korhonen A, Pölkki T. Factors Influencing Parental Participation in Neonatal Pain Alleviation. J Pediatr Nurs. Epub ahead of print 2016. DOI: 10.1016/j.pedn.2016.05.004.

6. Cruz MD, Fernandes AM, Oliveira CR. Epidemiology of painful procedures performed in neonates: A systematic review of observational studies. Eur J Pain (United Kingdom) 2015; 20: 489498.

7. Khoza S, Tjale A. Knowledge, attitudes and practices of neonatal staff concerning neonatal pain management. Curationis 2014; 37: 1-10.
8. Van De Velde M, De Buck F. Fetal and maternal analgesia/anesthesia for fetal procedures. Fetal Diagn Ther 2012; 31: 201-209.

9. Parry S. Acute pain management in the neonate. Anaesth Intensive Care Med 2014; 15: 111-115.

10. Herr K, Coyne PJ,McCaffery M, etal. Pain Assessment in the Patient Unable to Self-Report: Position Statement with Clinical Practice Recommendations. Pain Manag Nurs 2011; 12: 230-250.

11. Mehrnoush N, Ashktorab T, Heidarzadeh M, et al. Factors Influencing Neonatal Pain Management from the Perspectives of Nurses and Physicians in a Neonatal Intensive Care Unit: A Qualitative Study. Iran J Pediatr 2017; 28: 1-9.

12. Akuma AO, Jordan S. Pain management in neonates: A survey of nurses and doctors. J Adv Nurs 2012; 68: 1288-1301.

13. Sujatha S, Samson R. Nurses Knowledge and Neonatal pain management. Int J Adv Res 2015; 3: 732-737.

14. Ufashingabire CM, Nsereko E, Njunwa KJ, et al. Knowledge and attitudes of nurses regarding pain in the intensive care unit patients in Rwanda. Rwanda J Ser F Med Heal Sci 2016; 3: 21-26.

15. English M, Irimu G, Nyamai R, et al. Developing guidelines in low-income and middle-income countries: lessons from Kenya. Arch Dis Child 2017; 102: 846-851.

16. Cong X, Delaney C, Vazquez V. Neonatal nurses' perceptions of pain assessment and management in NICUs: a national survey. Adv Neonatal Care 2013; 13: 353-60.

17. Costa T, Rossato LM, Bueno M, et al. Nurses ' knowledge and practices regarding pain management in newborns. Rev Esc Enferm USP 2017; 51: 1-7.

18. McDonald RP, Ho MHR. Principles and practice in reporting structural equation analyses. Psychol Methods 2002; 7: 64-82.

19. Carbajal R, Rousset A, Danan C, et al. Epidemiology and Treatment of Painful Procedures in Neonates in Intensive Care Units. JAMA; 300. Epub ahead of print 2008. DOI: 10.1001/jama.300.1.60.

20. Cong X, McGrath JM, Delaney C, et al. Neonatal nurses' perceptions of pain management: Survey of the United States and China. Pain Manag Nurs 2014; 15: 834-844. 
21. Asadi-noghabi F, Tavassoli-farahi M, Yousefi $\mathrm{H}$, et al. Neonate Pain Management: What do Nurses Really Know? Glob J Health Sci 2014; 6: 284-293.

22. Abdel Razeq NM, Akuma AO, Jordan S. Status of Neonatal Pain Assessment and Management in Jordan. Pain Manag Nurs 2016; 17: 239-248.

23. Marchant A. 'Neonates do not feel pain': A critical review of the evidence. Biosci Horizons 2014; 7: 1-9.

24. Hall R., Anand KJ. Pain Management in Newborns. Clin Perinatol 2015; 41: 895-924.

25. Khoza SLT. The knowledge, attitude and beliefs of doctors and nurses concerning neonatal. University of the Witwatersrand, 2012.

26. Hossain S, Wiroonpanich W, Orapiriyakul R. Nurses' knowledge and attitudes in pain management practice of Post-Operative Children in Bangladesh. 2014; 1-12.

27. Land CA, Ali-advisor N. Nurses 'knowledge on pain management practices and organisational pain management policy a research paper submitted to the graduate school in partial fulfilment of the requirements for the degree masters of science in nursing by Ball State University MU.
28. Kopf A.; Patel N. Guide to Pain Management in Low-Resource Settings. Int Assoc study pain.

29. Stevens B, Yamada J, Ohlsson A, et al. Sucrose for analgesia in newborn infants undergoing painful procedures ( Review ). Cochrane Database Syst Rev. Epub ahead of print 2016. DOI: 10.1002/14651858. CD001069.pub5.www.cochranelibrary.com.

30. Chien-Yun D, Wan-Fei C, Yu-Hsi Y, et al. A Study on Modification of Knowledge, Attitude and Practice on Vocational High School Electronics Courses Integrated with Nanotechnology Concept. Int J Therm Environ Eng 2011; 4: 73-79.

31. Alzghoul BI, Abdullah NAC. Pain Management Practices by Nurses: An Application of the Knowledge, Attitude and Practices (KAP) Model. Glob J Health Sci 2015; 8: 154.

32. American Academy of Pediatrics. Prevention and Management of Procedural Pain in the Neonate: An Update. Pediatrics 2016; 137: e20154271-e20154271. 